Rapid Reviews COVID-19

\title{
Review 1: "Protein \\ arginylation is regulated \\ during SARS-CoV-2 \\ infection"
}

Fangliang Zhang ${ }^{1}$

1"University of Miami Miller School of Medicine: University of Miami School of Medicine Pharmacology UNITED STATES"

Published on: Jan 26, 2022

License: Creative Commons Attribution 4.0 International License (CC-BY 4.0). 


\section{$\underline{\text { RR:C19 Evidence Scale rating by reviewer: }}$}

- Potentially informative. The main claims made are not strongly justified by the methods and data, but may yield some insight. The results and conclusions of the study may resemble those from the hypothetical ideal study, but there is substantial room for doubt. Decision-makers should consider this evidence only with a thorough understanding of its weaknesses, alongside other evidence and theory. Decisionmakers should not consider this actionable, unless the weaknesses are clearly understood and there is other theory and evidence to further support it.

$* * * * * * * * * * * * * * * * * * * * * * * * * * * * * * * * * * * * * * *$

\section{Review:}

In my opinion, the RR:C19 Strength of Evidence Scale for this manuscript falls right between "overall reliable" and "Potentially informative".

Post-translational protein arginylation, which is mediated by arginyltransferase 1 (ATE1) in mammals, is an evolutionarily conserved but less-explored phenomenon. While the role of arginylation in embryo development and cancer progression is starting to gain attention, almost nothing is known about its involvement in infectious diseases. This manuscript, entitled "Protein arginylation is regulated during SARS-CoV2 infection", is among one of the first to report such a scenario. Facing challenges due to the general lack of research tools for a study involving arginylation, the authors made a plausible effort by using multiple approaches-including bioinformatics and immunoblots-to pinch the evidence together and show the changes of ATE1 and arginylation activity during SARS-CoV-2 infection. Such a strategy increased the overall reliability of the main conclusion, which describes a likely involvement of arginylation in response to the SARS-CoV-2 virus. Multiple pieces of the presented information can be considered inspiring or even fascinating. For example, the authors showed that two arginylation inhibitors, merbromin and tannic acid, possess the potential to suppress the proliferation and/or entry of the SARS-CoV-2 virus. This is surprisingly consistent with the results of another publication (doi.org/10.1128/AAC.00900-20), in which a potent suppression effect on SARS-CoV-2 was found for suramin. While unaware by many people including the original authors of the above paper, suramin is also a potent inhibitor of arginylation (doi: 10.1016/j.bcp.2012.01.012). In this sense, this current manuscript contains potentially 
high significance and novelty that may be important for the management of the COVID19 disease.

However, the manuscript at this stage contains significant deficiencies that negatively affect the quality of the data. As such, many details of the summary statement (abstract) cannot be considered fully reliable or be taken by the face value. The biggest concerns are in the quantification of Western Blots, where several apparent discrepancies exist between the quantified results and the immunoblots. For example, in Fig 6A, the ratio of ATE1/GAPDH in lanes 3 and 4 appear to be almost three folds more than lane 1 in the chart, while in the displayed blot such a difference cannot be seen - particularly when GAPDH loading is considered. Similarly, the ratio of BIPARG/BIP showed a significant dip in lane 7 according to the immunoblot, while in the chart, the quantified value appears to be quite high. Furthermore, the authors did not clarify an important detail in quantification regarding how they combine data points from multiple measurements. Such measurements are usually done on different immunoblots with separate antibody incubation and image acquisitions. As such, these data points cannot simply be combined without any cross-group normalization. It is unclear if, or how, the authors performed this important step in the quantification process. A reasonable and simple approach would be appointing a control sample (such as the untreated cells) as a normalization point and assuming it remains unchanged in different experiments (set at 1.0 or whatever arbitrary value). In addition, many of the comparisons are made between data points with drastic differences in loading amounts. This likely creates a problem for the reliability of the data because, based on our own experience, the linearity of a chemiluminescent assay is never $100 \%$. As such, the comparison of ratios should be performed when the "loading" or "divider" values are as close as possible. This is particularly a concern in Fig. 7C for the datapoints of ATE1/GAPDH and ACTB-ARG/ACTB.

Finally, some misinterpretation of the literature was found throughout the manuscript regarding the field of arginylation. For example, on Page 3, lines 11-12, the authors appear to state that all amino acids in the $\mathrm{N}$-end, except methionine, result in rapid degradation. However, the original study shows only charged or bulky amino acids have such effects (DOI: 10.1126/science.3018930). Also, on Page 3, line 24, the authors cited the study by Deka et al. (REF40) for the role of arginylation in promoting cell death, but this should be Kumar et al (REF39). Deka et al. actually showed an opposite effect of arginylation during the specific scenario of heat-shock priming. These can be fixed by rewriting. 